\title{
Studies on cyclic AMP in different compartments of cerebrospinal fluid
}

\author{
D. TSANG ${ }^{1}$, S. LAL, T. L. SOURKES, R. M. FORD, AND A. ARONOFF \\ From the Departments of Psychiatry, Queen Mary Veterans' Hospital, Montreal General Hospital and \\ McGill University, and the Department of Neurosurgery, Montreal General Hospital, \\ and the Department of Medicine, Queen Mary Veterans' Hospital, Montreal, Quebec, Canada
}

SYNOPSIS Adenosine 3', 5'-monophosphate (cAMP) was measured in the CSF of 42 patients undergoing radiological investigation, neurosurgical procedures, or investigation of hepatic coma. The concentration of cAMP was significantly higher in ventricular CSF than in lumbar CSF. Premedication with pentobarbitone plus promethazine increased cAMP in lumbar CSF. There was no difference in cAMP concentration in lumbar CSF obtained before or after injection of air or after the administration of diazepam during lumbar pneumoencephalography. Lumbar CSF cAMP cono centration was significantly increased in patients in hepatic coma. The concentration of cAMP in the lateral ventricle was not affected by general anaesthesia or by the presence of a complete block of the aqueduct of Sylvius. There was no decrease in lumbar CSF cAMP in patients with a complete stenosi of the aqueduct of Sylvius, partial blocks of CSF flow at the cervical level, or a complete block at the lower thoracic level. The concentration of cisternal CSF cAMP was similar to that of lumbar CSF命 These results suggest that (1) there is a ventriculolumbar gradient in the concentration of cAMP but of insufficient magnitude to be detected by mixing of lumbar and ventricular CSF during pneumoence phalography, (2) lumbar CSF cAMP concentration is not dependent on brain as a source of this nucleotide; the source of this nucleotide may be largely derived from the spinal cord, (3) premedicatio may affect the concentration of cAMP in lumbar CSF cAMP, (4) the formation of cAMP is unimpaire in hepatic coma.

Adenosine 3', 5'-monophosphate (cAMP) may play a role in the mediation of monoaminergic function in the central nervous system (Rall, 1972). In view of known derangements in monoaminergic function in Parkinson's disease (Hornykiewicz, 1971), postulated abnormalities in affective psychoses (Schildkraut, 1974), and changes in urinary excretion of cAMP in manic-depressive illness (Paul et al., 1971; Abdulla and Hamadah, 1970), various authors have looked for alterations in cAMP concentration in lumbar cerebrospinal fluid (LCSF) in various neurological (Cramer et al., 1973) and psychiatric disorders (Robison et al., 1970; Cramer et al., 1972a). Unfortunately, little information is available on the relative contribution of brain and spinal cord to the concentration of CAMP in LCSF so that interpreta-

${ }^{1}$ Address for correspondence and reprint requests: D. Tsang, Department of Psychiatry, Montreal General Hospital, Montreal H3G 1A4, P.Q., Canada.

(Accepted 7 July 1976.) tion of results based on LCSF findings must be made with caution.

In the present study we have investigated the source of CAMP in LCSF by measuring the concentration of this cyclic nucleotide in (1) different CSF compartments, (2) LCSF obtained before and after injection of air in patients undergoing lumbar pneumoencephalography (LPEG), and (3) CSF in patients with blocks in CSF flow. Also, in view of the belief that monoaminergic neurotransmitter function may be impaired in hepatic coma (Fischer and Baldessarini, 1971; Baldessarini and Fischer, 1973), we have measured the concentration of CAMP in LCSF in patients with this condition.

\section{METHODS}

Cyclic AMP was measured in CSF of 42 patients who were undergoing radiological investigation, neuro- 
surgical procedures, or investigation of hepatic coma (Table).

Two millilitres of CSF were obtained from the lateral ventricle in six patients (1-6) (three males and three females aged 40-62 years) during neurosurgical procedures. In four of the subjects (1-4) the CSF was obtained under general anaesthesia during revision of a ventriculoperitoneal shunt. CSF was also obtained under local anaesthesia in subjects 4 and 5. Patient 6 (a 47 year old male) had complete stenosis of the aqueduct, probably secondary to childhood meningitis. Ventricular CSF was obtained under local anaesthesia; three days before ventriculography CSF was obtained during LPEG.

Two millilitres of cisternal CSF were obtained in patient 7 who had a complete block in CSF flow at the level of T10 vertebra.

LCSF was obtained in 17 patients (8-24) who were undergoing LPEG (13 males and four females: aged 19-72 years). Two millilitres were obtained for cAMP determination before injection of air and also from the terminal volume of CSF removed after completion of the air studies. The amount of air injected was $57 \pm 4 \mathrm{ml}$ (mean \pm standard error of the mean) with a range of $30-95 \mathrm{ml}$. All subjects undergoing LPEG received premedication which in most cases consisted of pentobarbitone $(100 \mathrm{mg})$ plus promethazine $(50 \mathrm{mg})(\mathrm{n}=11)$. Five other patients received codeine $(60 \mathrm{mg})$ in addition to pento- barbitone plus promethazine. All premedication was administered intramuscularly one hour before the procedure was begun. Seven of the patients received intravenous diazepam $(5-20 \mathrm{mg})$ after the initial sample of CSF was taken. The diazepam was given 20-50 minutes before the final sample of CSF was withdrawn. Moderate cerebral atrophy was present in six of the subjects and mild atrophy in four. A completely normal LPEG was found in three individuals.

Two millilitres of LCSF were obtained during myelography in patients $25-38$ (seven males and seven females aged 33-75 years) before injection of the contrast medium. Myelography revealed either normal findings or evidence of lumbar or cervical disc protrusion without significant obstruction of the flow of contrast medium in patients 25-35 inclusive. Patients 36 and 37 had severe spinal cord compression with paraplegia secondary to cervical spondylosis. Patient 36 was suffering from dystonia musculorum deformans. Partial obstruction to CSF flow was present at $\mathrm{C} 6-\mathrm{C} 7$ intervertebral level in patient 36 and between $\mathrm{C} 4-\mathrm{C} 7$ in patient 37 . Patient 38 had a rapidly developing cord compression over a two day period with complete paraplegia; myelography revealed a complete block of CSF flow at the upper border of T10 vertebra probably due to an extradural mass. None of the subjects undergoing

T A B LE

CONCENTRATION OF CAMP IN CSF

\begin{tabular}{|c|c|c|c|}
\hline \multirow[b]{2}{*}{$\begin{array}{l}\text { Patients } \\
\text { (no.) }\end{array}$} & \multirow{2}{*}{ Source of CSF and clinical data } & \multicolumn{2}{|c|}{$c A M P(n m o l / 1)$} \\
\hline & & $\bar{x} \pm S E M$ & Range \\
\hline $\begin{array}{l}1-5 \\
6\end{array}$ & $\begin{array}{l}\text { Lateral ventricle } \\
\text { Lateral ventricle. Complete stenosis of aqueduct of Sylvius } \\
\text { Lumbar CSF before injection of air* }\end{array}$ & $\begin{array}{l}22.6 \pm 1.2+ \\
20 \\
55\end{array}$ & $20-26$ \\
\hline 7 & Cisternal CSF. Complete block upper level $\mathrm{T} 10$ vertebra & 10 & \\
\hline $8-24$ & $\begin{array}{l}\text { Lumbar CSF before injection of air* } \\
\text { Lumbar CSF after injection of air* }\end{array}$ & $\begin{array}{l}34.3 \pm 2.4 \S \\
34.7 \pm 2.3 \ddagger\end{array}$ & $\begin{array}{l}16-52 \\
20-52\end{array}$ \\
\hline $25-35$ & Lumbar CSF $\dagger$ & $13.7 \pm 1.4$ & $8-22$ \\
\hline 36 & Lumbar CSF†. Partial block C6-C7 & 12 & \\
\hline 37 & Lumbar CSF†. Partial block C4-C7 & 14 & \\
\hline 38 & Lumbar CSF†. Complete block upper level T 10 vertebra & 28 & \\
\hline $39-42$ & Lumbar CSF. Hepatic coma & $71.5 \pm 9.6^{\circ}$ & $60-100$ \\
\hline
\end{tabular}

* CSF obtained during lumbar pneumoencephalography (all subjects premedicated).

† CSF obtained during myelography (no premedication).

† Significantly greater than lumbar CSF obtained during myelography $(\mathrm{P}<0.001)$.

$\S$ Significantly greater than lumbar CSF obtained during myelography $(\mathrm{P}<0.001)$ and CSF from the lateral ventricles $(\mathrm{P}<0.02)$. Concentration of cAMP before and after air injection not significantly different.

ii Significantly greater than ventricular CSF or lumbar CSF obtained either at pneumoencephalography or myelography ( $P<0.001)$. 
myelography (or cisternal puncture) received premedication.

Patients 39-42 were male subjects aged 51-60 years with portal cirrhosis and in hepatic coma. One of the patients was in stage $2+$, one in stage $5+$, and the remaining two in stage $6+$ coma on a modified Webster-Davidson Scale (Lal et al., 1974) at the time of LCSF sampling. Three of the patients were on antibiotic therapy with or without lactulose; the fourth patient in stage $6+$ coma was on no medication at the time of lumbar puncture.

Samples of CSF were collected in ice cold tubes and immediately frozen and stored at $-20^{\circ} \mathrm{C}$ until assayed (Tsang et al., 1972).

Student's $t$ test and the Pearson product moment correlation coefficient $(r)$ were used in the statistical analysis of data (Ferguson, 1966).

\section{RESULTS}

The concentration of cAMP in CSF obtained from the lateral ventricle from patients $1-5$ was $22.6 \pm 1.2$ $\mathrm{nmol} / \mathrm{l}$ (Table). In the four subjects who were under general anaesthesia the range was $20-26 \mathrm{nmol} / \mathrm{l}$. In one of the subjects the concentration of cAMP in ventricular CSF was $20 \mathrm{nmol} / \mathrm{l}$ both under general anaesthesia and when obtained under local anaesthesia. In patient 5 the concentration of this nucleotide obtained under local anaesthesia was $22 \mathrm{nmol} / \mathrm{l}$.

The concentration of cAMP in ventricular CSF was significantly greater than that in LCSF obtained during myelography, but significantly less than that obtained during LPEG.

All subjects undergoing LPEG, but not those undergoing myelography or cisternography, received premedication. The concentration of cAMP in subjects undergoing LPEG was significantly higher than in LCSF obtained from patients undergoing myelography. In those subjects receiving premedication with pentobarbitone plus promethazine the concentration of cAMP was $36.3 \pm 2.3 \mathrm{nmol} / 1(n=11)$ and in those receiving codeine in addition $29.6 \pm 6.5$ $\mathrm{nmol} / \mathrm{l}(\mathrm{n}=5)(\mathrm{P}=\mathrm{NS})$. The concentrations of cAMP before and after introduction of air were not significantly different.

In patient 6 the presence of a complete stenosis of the aqueduct of Sylvius had no effect on the concentration of cAMP in the lateral ventricle. The concentration of CAMP in LCSF in this same patient obtained during LPEG three days kefore the ventricular tap was slightly above the range obtained in patients during LPEG who were without a block in CSF flow. The concentration of cAMP in cisternal CSF in patient 10 who had a complete block of CSF flow at the upper level of T10 vertebra and in subjects 36 and 37 who had partial restriction in CSF flow at the cervical level were within the range of values for LCSF obtained at myelography in patients without impairment of CSF flow.

In patient 38 who had a complete block of CSF flow at the upper level of T10 vertebra, the concentration of CAMP in LCSF was slightly above the range of values for subjects similarly undergoing myelography but without a block.

In the four patients in hepatic coma the concentration of cAMP in LCSF was significantly higher than that found in ventricular fluid or in LCSF whether obtained during myelography or LPEG.

There was no correlation between age and concentration of CAMP in LCSF in patients undergoing LPEG $(\mathrm{r}=+0.14 ; \mathrm{df}=15 ; \quad \mathrm{P}=\mathrm{NS})$ or myelography $(r=-0.09 ; d f=9 ; P=N S)$. Gender had no effect on the concentration of cAMP in patients examined by LPEG (men: $33.5 \pm 2.1 \mathrm{nmol} / 1$, $\mathrm{n}=13$; women: $37.8 \pm 3.1 \mathrm{nmol} / \mathrm{l}, \mathrm{n}=4)$ or myelography (men: $16.3 \pm 1.9 \mathrm{nmol} / \mathrm{l}, \mathrm{n}=5$; women: $11.6 \pm 1.6 \mathrm{nmol} / \mathrm{l}, \mathrm{n}=6$ ). There was no difference in cAMP concentration in patients with cerebrak atrophy $(34.3 \pm 2.1 \mathrm{nmol} / 1, \mathrm{n}=10)$ and those withoutes $(34.7 \pm 3.3 \mathrm{nmol} / 1, \mathrm{n}=7)$. This absence of difference is evident even if only those with significant cerebrab atrophy $(35.3 \pm 3.6 \mathrm{nmol} / 1, \mathrm{n}=6)$ are compared with those without atrophy.

Administration of diazepam to patients during LPEG had no effect on cAMP concentration (before $36.7 \pm 4.5 \mathrm{nmol} / 1$; after: $33.9 \pm 2.6 \mathrm{nmol} / \mathrm{l}$ ).

\section{DISCUSSION}

Previous authors have reported values for the concentration of CAMP in LCSF similar to our findings observed in patients undergoing myelography. Thus, Cramer et al. (1973), who examined LCSF in patients with various neurological disorders, central or peripheral, found a range of 6-24 nmol/1 (mean 13 \pm 0.71 ) and Robisan et al. (1970), in assorted neurological cases a mean of $16.2 \mathrm{nmol} / \mathrm{l}$.

In LCSF from patients undergoing LPEG the mean concentration of CAMP was 2.5 times that of LCSF obtained from patients undergoing myelography. One possible reason for the difference could be the difference in patient population undergoing the separate radiological procedures. Several of our patients undergoing LPEG had cerebral atrophy which has been reported to alter cAMP levels in LCSF (Myllylä et al., 1974). However, in contrast with Myllylä et al. (1974) who found a decrease in LCSF in patients with cerebral atrophy, we found no change. Increases in LCSF cAMP have been reported in acute cerebral infarction (Welch et al., 1975), acute meningitis (Heikkinen et al., 1975), within three days of an epileptic attack (Myllylä et al., 
1975a) and in patients suffering from acute or rapidly progressive damage to the central nervous system (Myllylä et al., 1975b). Our patients, in general, did not conform to these categories of conditions. Further, Cramer et al. (1973), found no change in LCSF cAMP in chronic neurological disorders.

A second possibility for the difference in cAMP concentration in LCSF in patients undergoing myelography and LPEG is that premedication increases CAMP. In this regard, certain peripherally administered drugs have been shown to increase cAMP in rabbit cisternal CSF (Sebens and Korf, 1975). In the present study either pentobarbitone or promethazine may have been responsible for the raised levels of cAMP. The former drug has been shown to increase cAMP in rat brain (Kimura et al., 1974) but promethazine, an antihistaminic phenothiazine, has a weak inhibitory effect on adenyl cyclase activity in brain (Uzunov and Weiss, 1972), which would tend to decrease the concentration of this nucleotide. It is possible that either or both these drugs affect the efflux of cAMP from CSF to blood, as is believed to be the case with probenecid (Cramer et al., 1972b), and thus increase cAMP in lumbar CSF.

Diazepam which increases cAMP in brain (Schultz, 1974) had no effect on LCSF cAMP when administered during LPEG. The interval between drug administration and sampling of CSF may have been too short to detect changes.

The concentration of cAMP in CSF from the lateral ventricle was similar whether obtained under general or local anaesthesia. Ventricular CSF cAMP concentration was significantly higher than in LCSF obtained at myelography. This difference points to the presence of a ventriculolumbar gradient in the concentration of this nucleotide. Despite this gradient the presence of complete stenosis of the aqueduct of Sylvius had no effect on the cAMP concentration in the lateral ventricles or an appreciable influence on the concentration of this nucleotide in LCSF. These observations indicate that the presence of cAMP in LCSF is not dependent on an input from the lateral ventricles. Also, adequate efflux of this nucleotide occurs at the level of the lateral ventricles.

The concentration of cAMP in cisternal CSF in the patient with a complete block at T10 vertebra was within the range of LCSF cAMP concentrations obtained during myelography. Presumably flow patterns of CSF and uptake patterns of this nucleotide preclude significant mixing of ventricular CSF cAMP and cisternal CSF cAMP.

Partial blocks in CSF flow at the cervical level had no effect on LCSF concentration of cAMP below the block. In the patient with a complete block in
CSF flow at the upper level of T10 vertebra the concentration of cAMP below the block was above the range of values for subjects examined by myelography without obstruction. These data suggest that brain need not be the source of cAMP in LCSF and that the major source of origin may be the spinal cord. It is possible, of course, that cAMP in LCSF is not derived only from brain and/or spinal cord but also from blood. In this regard, Sebens and Korf (1975) have shown that, in the rabbit, intravenously administered cAMP does not enter CSF. On the other hand, Dascombe and Milton (1975) have shown that cAMP of cat CSF increases after intravenous administration of this nucleotide.

The higher value in LCSF cAMP below the block at T10 vertebra in patient 38 compared with subjects with unrestricted flow in CSF may be related to effects of the block on diffusion or egress of cAMP from LCSF or changes in production and absorption of CSF below the block.

The magnitude of difference between ventricular and LCSF concentration of CAMP is sufficiently small that mixing of fluid from the two compartments, as occurs during LPEG, reveals no significant change in concentration of LCSF cAMP. This latter observation is in keeping with the observation of Myllylä et al. (1974) who also found no change in LCSF CAMP during LPEG and Cramer et al. (1972a) who found no effect of exercise on LCSF cAMP concentration. Both these procedures produce significant changes in monoamine acid catabolite concentrations (Post et al., 1973; Garelis and Sourkes, 1974) where significant gradients between ventricular and lumbar CSF exist. The absence of significant changes in LCSF cAMP concentration after LPEG is further evidence that brain is not the major source of LCSF cAMP. This does not exclude the possibility that, under certain conditions which result in marked release of cAMP from brain into ventricular CSF or damage to the transport mechanism of the nucleotide out of ventricular CSF, changes might be observed in LCSF. It seems, however, unlikely that postulated changes in brain cAMP metabolism in affective disorders could be reflected in changes in LCSF cAMP concentration unless the dysfunction exerted a widespread influence on central nervous system cAMP metabolism.

In hepatic coma, in which the presumed toxin ammonia (Walker and Schenker, 1970) has a widespread effect on the central nervous system, there was no decrease in cAMP concentration in LCSF as might have been expected if monoaminergic neural transmission were impaired. In fact, there was a striking increase in cAMP. This rise may indicate an increased synthesis, a decreased degradation, or an impaired elimination of this nucleotide from CSF. 
The authors thank Dr. R. Wee and Dr. J. D. Chan, of the Department of Neuroradiology, Montreal General Hospital, for their assistance in this study which was supported by grants from the Department of Veterans' Affairs and the Medical Research Council of Canada. Additional support was received from Merck Frosst Laboratories, Quebec, Canada. D. Tsang is a Fellow of the Quebec Medical Research Council.

\section{REFERENCES}

Abdulla, Y. H., and Hamadah, K. (1970). 3', 5' cyclic adenosine monophosphate in depression and mania. Lancet, 1, 378-381.

Baldessarini, R. J., and Fischer, J. E. (1973). Serotonin metabolism in rat brain after surgical diversion of the portal venous circulation. Nature New Biology, 245, 25-27.

Cramer, H., Goodwin, F. K., Post, R. M., and Bunney, W. E. (1972a). Effects of probenecid on cerebrospinal fluid cyclic A.M.P. in affective illness. Lancet, 1, 1346-1347.

Cramer, H., Ng, L. K. Y., and Chase, T. N. (1972b). Effect of probenecid on levels of cyclic AMP in human cerebrospinal fluid. Journal of Neurochemistry, 19, 1601-1602.

Cramer, H., Ng, L. K. Y., and Chase, T. N. (1973). Adenosine 3', 5'-monophosphate in cerebrospinal fluid. Effect of drugs and neurologic disease. Archives of Neurology (Chic.), 29, 197-199.

Dascombe, M. J., and Milton, A. S. (1975). Cyclic adenosine 3', 5'-monophosphate in cerebrospinal fluid. British Journal of Pharmacology, 54, 254P-255P.

Ferguson, G. A. (1966). Statistical Analysis in Psychology and Education, 2nd edn. McGraw-Hill: Toronto.

Fischer, J. E., and Baldessarini, R. J. (1971). False neurotransmitters and hepatic failure. Lancet, 2, 75-80.

Garelis, E., and Sourkes, T. L. (1974). Use of cerebrospinal fluid drawn at pneumoencephalography in the study of monoamine metabolism in man. Journal of Neurology, Neurosurgery, and Psychiatry, 37, 704-710.

Heikkinen, E. R., Similä, S., Myllylä, V. V., Hokkanen, E., and Vapaatalo, H. (1975). Cyclic adenosine-3', 5'monophosphate concentration and enzyme activities of cerebrospinal fluid in meningitis of children. Zeitschrift für Kinderheilkunde, 120, 243-250.

Hornykiewicz, O. (1971). Neurochemical pathology and pharmacology of brain dopamine and acetylcholine: rational basis for the current drug treatment of Parkinsonism. In Recent Advances in Parkinson's Disease, pp. 33-65. Edited by F. H. McDowell and C. H. Markham. Davis: Philadelphia.

Kimura, H., Thomas, E., and Murad, F. (1974). Effects of decapitation, ether and pentobarbital on guanosine $3^{\prime}$, $5^{\prime}$-phosphate and adenosine $3^{\prime}, 5^{\prime}$-phosphate levels in rat tissues. Biochimica et Biophysica Acta, 343, 519-528.

Lal, S., Aronoff, A., Garelis, E., Sourkes, T. L., Young, S. N., and de la Vega, C. E. (1974). Cerebrospinal fluid homovanillic acid, 5-hydroxyindoleacetic acid, lactic acid, and $\mathrm{pH}$ before and after probenecid in hepatic coma. Clinical Neurology and Neurosurgery, 77, 142154.

Myllylä, V. V., Heikkinen, E. R., Similä, S., Hokkanen, E., and Vapaatalo, H. (1975b). Cerebrospinal fluid concentration and urinary excretion of cyclic adenosine- $3^{\prime}$, $5^{\prime}$-monophosphate in various diseases of children. Zeitschrift für Kinderheilkunde, 118, 259-264.

Myllylä, V. V., Heikkinen, E. R., Vapaatalo, H., and Hokkanen, E. (1975a). Cyclic AMP concentration and enzyme activities of cerebrospinal fluid in patients with epilepsy or central nervous system damage. European Neurology, 13, 123-130.

Myllylä, V. V., Vapaatalo, H., Hokkanen, E., and Heikkinen, E. R. (1974). Cerebrospinal fluid concentration of cyclic adenosine-3', 5'-monophosphate and pneumoencephalography. European Neurology, 12, 28-32.

Paul, M. I., Cramer, H., and Bunney, W. E. (1971) Urinary adenosine $3^{\prime}, 5^{\prime}$-monophosphate in the switch process from depression to mania. Science, 171, 300 303.

Post, R. M., Kotin, J., Goodwin, F. K., and Gordon, E. K. (1973). Psychomotor activity and cerebrospinal fluid amine metabolites in affective illness. American Journal of Psychiatry, 130, 67-72.

Robison, G. A., Coppen, A. J., Whybrow, P. C., and Prange, A. J. (1970). Cyclic AMP in affective disorders Lancet, 2, 1028-1029.

Rall, T. W. (1972). Role of adenosine 3', 5'-monophosphate (cyclic AMP) in actions of catecholamines Pharmacological Reviews, 24, 399-409.

Schildkraut, J. J. (1974). Biogenic amines and affective? disorders. Annual Review of Medicine, 25, 333-348.

Schultz, J. (1974). Adenosine 3', 5'-monophosphate in guinea pig cerebral cortical slices: effect of benzodiazepines. Journal of Neurochemistry, 22, 685-690.

Sebens, J. B., and Korf, J. (1975). Cyclic AMP in cerebrospinal fluid: accumulation following probenecid and biogenic amines. Experimental Neurology, 46, 333-344

Tsang, C. P. W., Lehotay, D. C., and Murphy, B. E. P. (1972). Competitive binding assay for adenosine $3^{\prime}$, 5'-monophosphate employing a bovine adrenal protein: application to urine, plasma and tissues. Journal of Clinical Endocrinology and Metabolism, 35, 809-817.

Uzunov, P., and Weiss, B. (1972). Psychopharmacological agents and the cyclic AMP system of rat brain. Advances in Cyclic Nucleotide Research, 1, 435-452.

Walker, C. O., and Schenker, S. (1970). Pathogenesis of hepatic encephalopathy with special reference to the role of ammonia. American Journal of Clinical Nutrition, 26, 619-632.

Welch, K. M. A., Meyer, J. S., and Chee, A. N. C. (1975) Evidence for disordered cyclic AMP metabolism in patients with cerebral infarction. European Neurology, 13, 144-154. 